http://dx.doi.org/10.11646/zootaxa.3794.4.8

http://zoobank.org/urn:lsid:zoobank.org:pub:C23C4B9E-26C7-465C-870E-7E8DFCA69EC5

\title{
A new subgenus and species of Topomyia (Diptera: Culicidae: Sabethini) based on a remarkable male mosquito from Sabah, Malaysia
}

\author{
RALPH E. HARBACH \& C. LORNA CULVERWELL \\ Department of Life Sciences, Natural History Museum, Cromwell Road, London SW7 5BD, UK. \\ E-mail:r.harbach@nhm.ac.uk; l.culverwell@nhm.ac.uk
}

\begin{abstract}
Miyagiella Harbach, subgen. nov., is introduced as a new subgenus of Topomyia Leicester for a remarkable male mosquito, Topomyia discors Harbach, sp. nov., from Sabah, Malaysia. A diagnosis of the subgenus is provided that features unique anatomical characters of the genitalia of the holotype male. Miyagiella is very distinct from the two previously recognised subgenera of Topomyia, but is perhaps more closely related to the nominotypical subgenus than to subgenus Suaymyia Thurman. Salient differences that distinguish the three subgenera are contrasted; the holotype male of To. discors is described and its unique genitalia are illustrated.
\end{abstract}

Key words: Mosquitoes, Miyagiella, new subgenus, taxonomy, Topomyia discors sp. nov.

\section{Introduction}

The new subgenus and species named and described here are based on a unique male mosquito discovered among other mosquitoes collected in a light trap hung at the edge of a small stand of bamboo in northern Sabah of Malaysian Borneo. Other collections made at and in the vicinity of the site were examined for additional specimens, but none were found. The genitalia of the single specimen are so remarkably different than those of all other species of Topomyia Leicester that the species cannot be placed in either of the two currently recognised subgenera, Suaymyia Thurman and Topomyia sensu stricto, which are based on features of the forelegs and genitalia of males (Thurman, 1959).

\section{Materials and methods}

This study is based on a single male mosquito captured in an un-baited CDC light trap (see Holotype below). The specimen was point-mounted on an insect pin and its genitalia were dissected, cleared in $5 \% \mathrm{NaOH}$ for $2 \mathrm{~h}$ at $50^{\circ} \mathrm{C}$ and mounted in Euparal on a microscope slide. The pinned specimen was examined under simulated natural light with an Olympus SZ6045 stereomicroscope. The dissected genitalia were studied and illustrated with an Olympus BX50 compound microscope fitted with differential interference contrast optics and a camera lucida. Digital images of the genitalia were taken with a Canon 550D digital camera mounted on a Leica M125 stereomicroscope; Helicon Focus version 3.03 software (Helicon Soft Ltd, Kharkov, Ukraine) was used to obtain extended-focus images. Anatomical terminology and abbreviations used in the descriptions and illustrations, respectively, follow Harbach \& Knight (1980, 1982), revised and updated by Harbach (2014). 


\section{Taxonomy}

\section{Topomyia subgenus Miyagiella Harbach, subgen. nov.}

Type species: Topomyia (Miyagiella) discors Harbach, sp. nov.

Diagnosis. Known only from the holotype male (see below); distinguished from subgenera Suaymyia Thurman and Topomyia Leicester by combinations of the following characters, which are contrasted in Table 1. Foretarsomere 2 longer than tarsomere 3; foreungues unequal, larger (anterior) foreunguis with post-basal tooth; gonocoxite with few inconspicuous narrow scales, gonostylus attached subapically on mesal surface, gonostylar claw absent; sternum IX without scales (setae present); tergum IX broad, without setae; claspette with ventral lobe, no dorsal lobe; aedeagus very large, bulbous; tergum $\mathrm{X}$ and paraprocts fused to inner posterior margin of tergum IX; tergum $\mathrm{X}$ narrow, strip-like; paraproct a stout apically blunt process without teeth; cercal setae absent.

Etymology. Miyagiella, a Latinized diminutive patronymic, is established here to honour Professor Dr Ichiro Miyagi for his many contributions to the taxonomy of Oriental mosquitoes, especially those of genus Topomyia. The subgeneric name is feminine. The three-letter abbreviation Myg. is recommended for the subgenus.

TABLE 1. Comparison of diagnostic and differential characters that distinguish the subgenera of genus Topomyia.

\begin{tabular}{llll}
\hline Character & Suaymyia & Topomyia & Miyagiella \\
\hline Foretarsomere 2 & Longer than tarsomere 3 & As long or shorter than tarsomere 3 & $\begin{array}{l}\text { Longer than tarsomere 3 } \\
\text { Ungues }\end{array}$ \\
Equal and simple in both sexes & Equal and simple in both sexes & $\begin{array}{l}\text { Larger foreunguis of male with } \\
\text { tooth }\end{array}$ \\
Gonocoxite & With conspicuous scales & With conspicuous scales among & Few inconspicuous scales among \\
among setae & setae & setae \\
Gonostylus & Apical & Apical (usually) or subapical* & Subapical \\
Gonostylar claw & Present & Present & Absent \\
Sternum IX & With scales and usually setae & With scales and setae & Without scales, setae only \\
Tergum IX & Narrow, with setae & Broad, with setae & Broad, without setae \\
Claspette & Dorsal lobe absent & Dorsal lobe present & Dorsal lobe absent \\
Aedeagus & Elongate & Elongate & Bulbous \\
Tergum X & Not evident & Not evident & Narrow sclerite joined to tergum IX \\
Paraproct & Independent slender sclerite & Independent elongate sclerite with & Stout process of tergum IX without \\
& with apical teeth & pointed apex & teeth \\
Cercal setae & Present/absent & Present & Absent \\
\hline
\end{tabular}

*Topomyia gracilis Leicester, To. hardini Miyagi, Toma \& Ramalingam, To. minor Leicester, To. pilosa Brug and To. winter Dong, Wu \& Mao.

\section{Topomyia (Miyagiella) discors Harbach, sp. nov.}

Adult. Holotype male. Largely denuded, missing left foreleg, left mid- and hindtarsomeres 2-5, right midleg and right hindtarsus; left wing crumpled, right wing damaged apically; scutellum and adjacent areas of scutum, mesopostnotum and right wing obscured by fungal hyphae. Head: Vertex with broad decumbent silvery scales, erect scales apparently absent; pair of stout dark interocular setae present; compound eyes contiguous above antennal pedicels; antenna $1.76 \mathrm{~mm}$, pedicel dark, bare, flagellum strongly verticillate, proximal flagellomeres without scales; proboscis and maxillary palpus entirely dark-scaled, proboscis $2.65 \mathrm{~mm}$, slightly bent ventrad in distal 0.5; palpus very short, about 0.11 length of proboscis. Thorax: Integument brown; scutum largely denuded, few pale piliform scales remain on anterior promontory, prescutellar area and scutellum obscured by fungal hyphae, mesopostnotum partly covered by hyphae but apparently without scales and setae, paratergite apparently bare; ante- and postpronota denuded, postpronotum with row of setal alveoli on posterior margin; thoracic pleura largely denuded, alveoli of 4 prespiracular setae visible, patch of lower mesokatepisternal setae and silvery spatulate scales present; upper mesepimeral setae and silvery spatulate scales also present. Wing: Dark-scaled; cell 
R2 about 1.4 times length of vein R2+3; anal vein ends beyond base of mediocubital crossvein. Halter: Scabellum and pedicel pale; capitellum with dark scales. Legs: Fore- and midcoxae with silver scales, hindcoxa with setae only, rest of legs dark-scaled except fore- and midfemora narrowly pale-scaled ventrally, proximal 0.8 of anteroventral surface of hindfemur pale-scaled, rest of legs dark-scaled; foretarsomere 3 distinctly shorter than tarsomere 2, 0.6 as long; foreungues unequal, larger anterior unguis with post-basal tooth. Abdomen: Terga apparently dark-scaled with indications of pale-scaled lateral margins; sterna apparently pale-scaled. Genitalia (Figs 1, 2): As illustrated. Tergum IX (Figs 1A, 2A) broad, without setae; sternum IX (Fig. 2E) very large, posterior margin produced medially as a short somewhat bi-lobed projection with 2 caudally directed peg-like setae on either side, setae on caudal 0.5 , no scales; gonocoxite short, obtuse, bulbous, setae on approximately distal 0.5 of dorsal and lateral surfaces, few inconspicuous narrow scales; mesal surface (Figs 1B, 2C) with a lumpy setose membranous tergomesal protuberance ( $=$ ? ventro-medial lobe of Thurman, 1959; dorsomesal lobe of Miyagi et al., 2012) and a heavily sclerotised anvil-like sternomesal lobe (= ? ventro-apical lobe of Thurman, 1959; apical lobe of Miyagi et al., 2012) bearing an apicolateral row of short sensilla basiconica; gonostylus (Figs 1, 2A-C) sicklelike, borne subapically, directed mesad, with line of setae on tergomesal margin and distally on ventral surface, gonostylar claw absent; claspette (ventral lobe) (Figs 1B, 2C) long, rod-like, borne tergomesally on gonocoxite at level of gonostylus, bearing 2 strong apical setae and a long lateral seta at mid-length, dorsal lobe absent; apex of gonocoxite with a very long, stout, sinuous, articulated spine (Figs 1, 2A-C); aedeagus (Figs 1A, 2A,D) very large, broad, width 0.8 length, widest at middle; composed of 2 complex lateral plates joined post-medially by transverse tergal bridge, caudal end of each plate with a flap-like apical tergal process and a sclerotised sternal crest, sternal surface of each plate with long submedian sternal arm that extends most of length of aedeagus, arms of opposite plates closely aligned and narrowly joined at mid-length, each joining thickened anterolateral margin of lateral plate; tergum $\mathrm{X}$ and paraprocts (Figs 1A, 2A) united with inner posterior margin of tergum IX, paraprocts short, stout, partially supporting basolateral margins of proctiger; proctiger large, membranous, cercal setae absent.
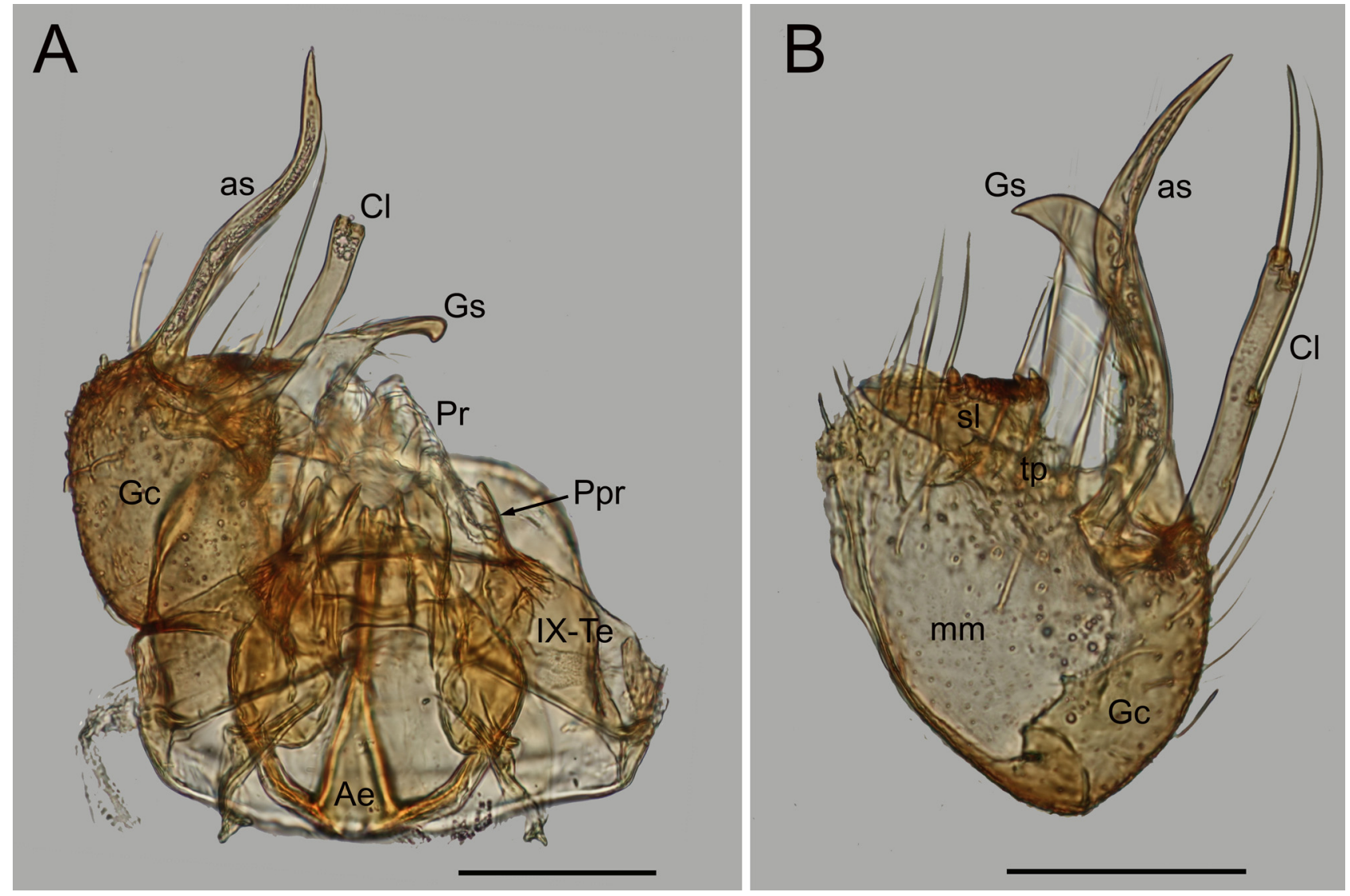

FIGURE 1. Focus stacking images of the male genitalia of Topomyia (Miyagiella) discors. A, Tergal view of the genitalia with the right gonocoxopodite removed. B, Mesal view of the dissected right gonocoxopodite. Scale bars $=0.15 \mathrm{~mm}$. Ae $=$ aedeagus; as = articulated spine; $\mathrm{Cl}=$ claspette; $\mathrm{Gc}=$ gonocoxite; $\mathrm{Gs}=$ gonostylus; $\mathrm{mm}=$ mesal membrane; $\mathrm{Ppr}=$ paraproct; $\mathrm{Pr}$ $=$ proctiger; $\mathrm{sl}=$ sternomesal lobe; $\mathrm{tp}=$ tergomesal protuberance; $\mathrm{IX}-\mathrm{Te}=$ tergum IX. 

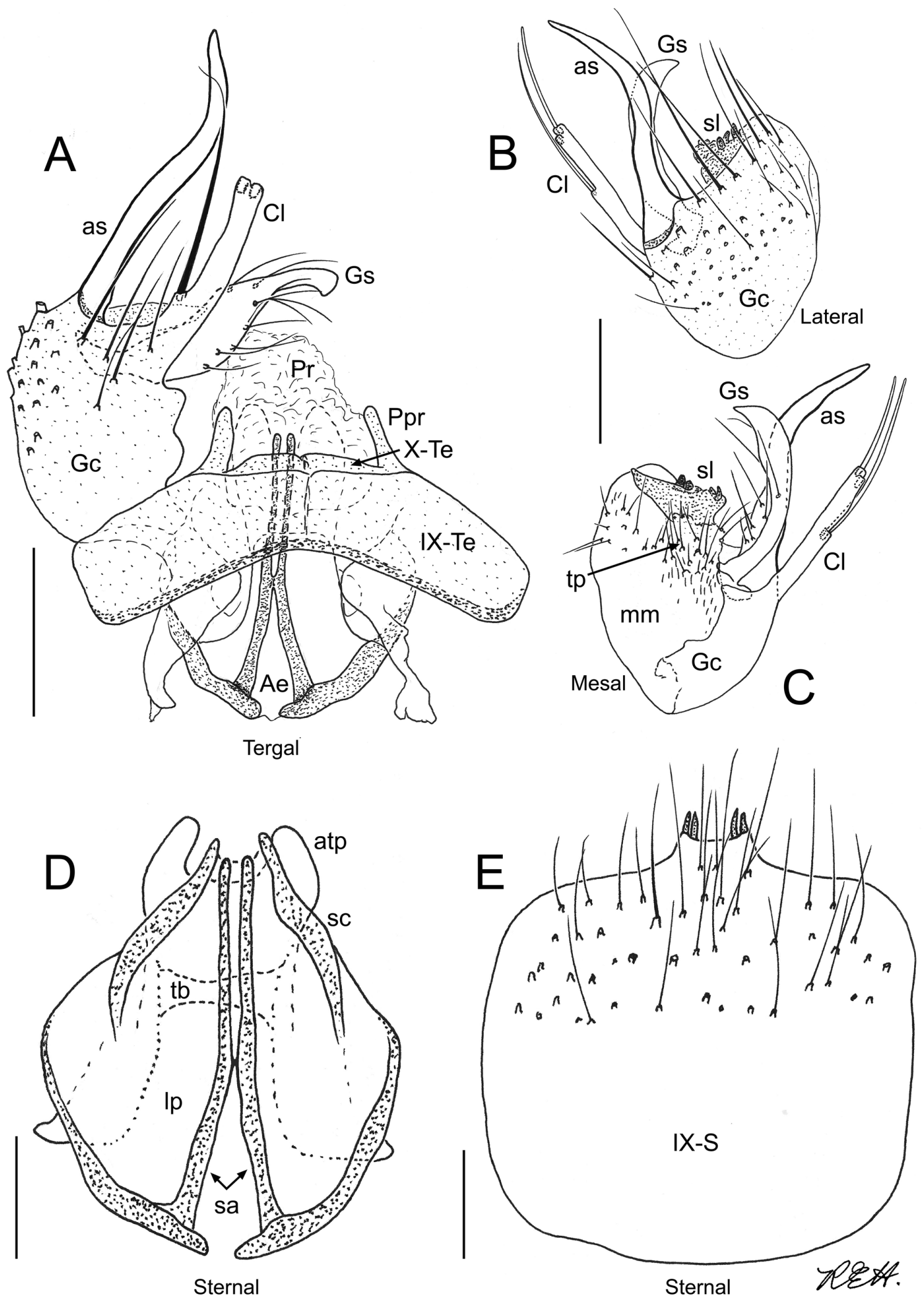

FIGURE 2. Drawings of the male genitalia of Topomyia (Miyagiella) discors (cf. Fig. 1). A, Genitalia with the right gonocoxopodite removed. B,C, Right gonocoxopodite. D, Aedeagus. E, Sternum IX. Aspects as indicated; scale bars $=0.15$ $\mathrm{mm}(\mathrm{A}, \mathrm{B}, \mathrm{C})$ and $0.075 \mathrm{~mm}(\mathrm{D}, \mathrm{E})$. Ae = aedeagus; as = articulated spine; atp = apical tergal process; $\mathrm{Cl}=\mathrm{claspette} ; \mathrm{Gc}=$ gonocoxite; $\mathrm{Gs}=$ gonostylus; $\mathrm{lp}=$ lateral plate; $\mathrm{mm}=$ mesal membrane; $\mathrm{Ppr}=$ paraproct; $\mathrm{Pr}=$ proctiger; $\mathrm{sa}=\mathrm{sternal}$ arm; $\mathrm{sc}=$ sternal crest; $\mathrm{sl}=$ sternomesal lobe; $\mathrm{tb}=$ tergal bridge; $\mathrm{tp}=$ tergomesal protuberance; IX-S = sternum IX; IX-Te = tergum IX; X$\mathrm{Te}=$ tergum $\mathrm{X}$. 
Female and immature stages. Unknown.

Etymology. The specific name is taken from the Latin single-termination third declension adjective discors ( $\mathrm{m}, \mathrm{f}, \mathrm{n}$; genitive discordis), meaning different or unlike, in reference to the unusual male genitalia.

Bionomics. The holotype was captured in a light trap at the edge of a stand of bamboo beside an agricultural field. As all species of Topomyia and other sabethine mosquitoes are associated with phytotelm habitats, it seems likely that the immature stages of To. discors may develop in bamboo internodes.

Distribution. Known only from the type locality in northern Sabah, Malaysia.

Holotype. Male, with dissected genitalia on microscope slide ( ${ }^{\lambda} \mathrm{G}$ Prep. 13/28), MALAYSIA: Sabah, Kampung Paradason, $06^{\circ} 46.135 \mathrm{~N} 116^{\circ} 47.183 \mathrm{~W}$, captured in non-baited CDC light trap hung $3.9 \mathrm{~m}$ above ground at edge of small stand of bamboo, 2.viii.2013 (S. Richi \& F. Hawkes). The specimen is deposited in the National History Museum (NHM), London.

\section{Discussion}

The holotype male of To. discors was listed as an "unidentifiable male mosquito in the study of Richi (2013). It is undoubtedly a species of Topomyia, but its exceptional morphological features preclude it from being placed in either of the two currently recognised subgenera, Suaymyia and Topomyia; hence, the recognition of a new subgenus for this unusual mosquito. The toothed anterior foreunguis, absence of setae on tergum IX, the columnar claspette (ventral lobe), the very broad, distinctive aedeagus and the absence of a gonostylar claw easily distinguish it from males of all other currently known species of Topomyia. The straight foretarsus with the second tarsomere longer than the third tarsomere also distinguishes To. discors from males of subgenus Topomyia, which have the distal tarsomeres elbowed and directed posteriorly.

It is noted that the structure referred to as a claspette in species of Topomyia may not be strictly homologous with the claspette, or basal mesal lobe, of other culicine mosquitoes. According to Thurman (1959), the ventral lobe of the claspette is fused with the ventral margin of the gonocoxite and is united with its mate by a narrow connection; the claspette of species of subgenus Topomyia also has a long, slender dorsal lobe, bearing a single filamentous apical seta, which is not fused to the gonocoxite. Topomyia discors differs in having the ventral lobe fused to the dorsal margin of the gonocoxite with no connection to the ventral margin or its mate on the opposite side. If the columnar structure in To. discors is homologous with the ventral lobe of other Topomyia, then its independent fusion with the dorsal surface of the gonocoxite is another unique feature of the species.

Of the known species of Topomyia, only To. (Top.) minor Leicester has a claspette that bears some resemblance to the claspette of To. discors. In addition to being longer than usual among species of subgenus Topomyia, the claspette of To. minor bears a very long, sinuous, basolateral, spine-like seta reminiscent of the much stouter articulated spine of To. discors. Although the claspette of To. minor is borne in the usual position at the base of the gonocoxite, it is conceivable that the spine-like seta is homologous with the articulated spine of To. discors, which assumes that the claspette of the latter species broke free of its mate, migrated dorsocaudad and became fused with the dorsal margin of the gonocoxite during the course of evolution. The gonostylus of To. minor, though markedly different, is attached subapically more or less as in To. discors. The claspette of To. nepenthicola Miyagi \& Toma of subgenus Suaymyia is a small setose lobe (characteristic of the subgenus), but is unusual in having a long, sinuous, basolateral, spine-like seta as in To. minor of the nominotypical subgenus.

Topomyia occur in the Oriental Region, with extensions into the southern Palaearctic (Ryukyu Archipelago) and the Australasian Region (Sulawesi, Maluku Islands, Biak Island and New Guinea). With the taxa described here, the genus includes 64 species in three subgenera, Miyagiella (1 species), Suaymyia (22 species) and Topomyia (41 species). Rattanarithikul et al. (2007) listed 23 species in Thailand, four unnamed and two without subgeneric placement. Topomyia is obviously a diverse assemblage of taxa, the discovery, identification and classification of which is far from complete. 


\section{Acknowledgements}

We are grateful to Sophie Richi and Frances Hawkes for allowing us to examine mosquitoes they collected during a collaborative research project conducted by the London School of Hygiene and Tropical Medicine (project leader Dr Chris Drakeley) that contained the unique mosquito described here. We are also grateful to Theresa Howard, Natural History Museum, London, for curatorial assistance.

\section{References}

Harbach, R.E. (2014) Mosquito Taxonomic Inventory. Available from: http://mosquito-taxonomic-inventory.info/ (accessed 4 March 2014)

Harbach, R.E. \& Knight, K.L. (1980) Taxonomists' glossary of mosquito anatomy. Plexus Publishing, Marlton, New Jersey, $415 \mathrm{pp}$.

Harbach, R.E. \& Knight, K.L. (1982) Corrections and additions to Taxonomists' glossary of mosquito anatomy. Mosquito Systematics (for 1981), 13, 201-217.

Miyagi, I., Toma, T., Okazawa, T., Wong, S.F., Leh, M.U. \& Yong, H.S. (2012) Three new phytotelma mosquitoes of the genus Topomyia (Diptera: Culicidae) from Katibas, Lanjak-Entimau, Sarawak, Malaysia. Journal of Science and Technology in the Tropics, 8, 97-117.

Rattanarithikul, R., Harbach, R.E., Harrison, B.A., Panthusiri, P. \& Coleman, R.E. (2007) Illustrated keys to the mosquitoes of Thailand. V. Genera Orthopodomyia, Kimia, Malaya, Topomyia, Tripteroides, and Toxorhynchites. Southeast Asian Journal of Tropical Medicine and Public Health, 38 (Suppl. 2), 1-65.

Richi, S. (2013) Bionomics of the Leucosphyrus group of Anopheline [sic] mosquitoes and experimentation with outdoor trapping techniques. MSc Biology \& Control of Disease Vectors, London School of Hygiene and Tropical Medicine, London, viii +39 pp.

Thurman, E.B. (1959) A contribution to a revision of the Culicidae of northern Thailand. University of Maryland Agricultural Experimental Station Bulletin, A-100, 1-182. 